\title{
Spinal cord injury in a child: a long term follow-up study. Case report
}

\author{
O Yoshimura $^{1}$, T Murakami ${ }^{2}$, M Kawamura $^{1}$ and K Takayanagi ${ }^{2}$ \\ ${ }^{1}$ Institute of Health Science, Hiroshima University 1-1-89 Higashisennda, Naka-ku, Hiroshima 730; ${ }^{2}$ Kyushu \\ College of Rehabilitation, 1-5-1 Kuzuhara-Takamatsu, Kokuraminami-ku, Kitakyushu 800-02, Japan
}

The main features of spinal cord injuries in children are known to be that (1) plain radiographs do not show the bony injury; (2) many of the injuries are complete spinal cord injuries; (3) many involve the upper thoracic spine; and (4) the duration of spinal shock is short. Complications such as pressure sores occur just as easily in children as in adults and the injuries are intractable. Typical complications in children with spinal cord injuries are spinal derformity and hip dislocation. We discuss a patient with a C7 spinal cord injury caused by a fall when the patient was 3 years old, and the physical complications occurring during the 15 years following the injury.

Keywords: child; spinal cord injury; complications

\section{Introduction}

Spinal cord injuries (SCI) in children usually show specific clinical phenomena that are not usually observed in adults as children are in the growing process. The level and type of the vertebral column injury and the likelihood of completeness of the neural injury are to be noted, also the duration of spinal shock. In addition, there are certain complications that may eventually develop as the child grows older. A report is made of a SCI in a child.

\section{Case report}

The patient was an 18-year-old senior high school boy who fell to the ground from a fourth floor window of his apartment house at the age of 3 years and sustained a serious SCI (Figure 1). Roentgenography of the cervical spine failed to reveal a fracture or dislocation as is so common in cases of SCI in children. There was a complete

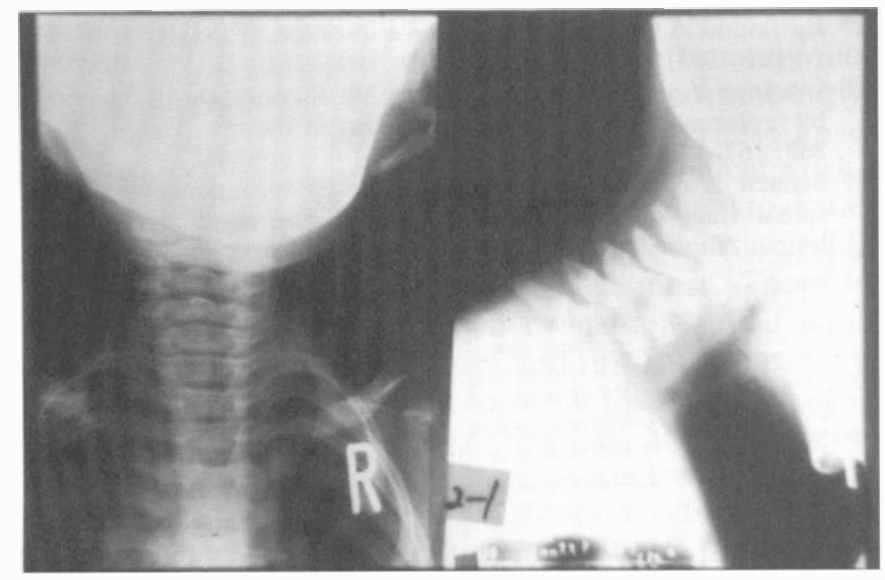

Figure 1 Simple roentgenography at the age of 3 revealed no sign of dislocation or fracture neural lesion at C7 with spasticity of moderate severity. Initially he had reflex incontinence, and he was able to do self-catheterisation after the age of 4 years. He had periodic renal function tests, pyelography and cystography, which were normal. He could drive a wheelchair and was discharged from hospital. Since then, he was followed up in hospital every 3 months as an outpatient and acetabular dysplasia and luxatio coxae became evident but no treatment was carried out in view of the complete C7 cord lesion. Without any particular trouble, he entered an elementary school at the age of 6 . He had some handicap with his fingers, but could use a pencil with his thumb and index finger by a lateral pinch sufficiently for writing speed and calligraphic balance. He entered a junior high school at the age of 12 . Spinal deformity appeared and worsened at the age of 14 (Figure 2); for its prophylaxis, a variety of treatments such as sheet sockets, spinal orthosis and therapeutic exercises were tried, but despite the presence of a severe spinal deformity, pelvic tilt and luxatio coxae, no surgical treatment was carried out. Because of fear of the occurrence of pressure sores due to the pelvic tilt and luxatio coxae, he was advised to do push-up exercises and to use the ROHO cushion. Pulmonary function test revealed $1,900 \mathrm{ml}$ vital capacity and $82 \%$ forced expiratory volume in $1 \mathrm{~s}$. He joined a personal computer club for extracurricular activities.

A recent MRI study (Figure 3) showed that the spinal cord at the C4-5 level was narrowed and gave a signal almost equal to the concentration of cerebrospinal fluid in this region. The bodies of the vertebrae of $\mathrm{C} 3-7$ were deformed and the intervertebral disc of C5-6 had disappeared; thus the spinal cord was deformed. Plain roentgenography taken at the age of 3 years had not shown evidence of dislocation or of a fracture, but it is presumed that he had sustained injuries at the end plates and the intervertebral disc at that time.

\section{Discussion}

The incidence of SCI in children is reported to be a few percent,${ }^{1-4}$ and $\mathrm{SCI}$ is uncommon in babies. 

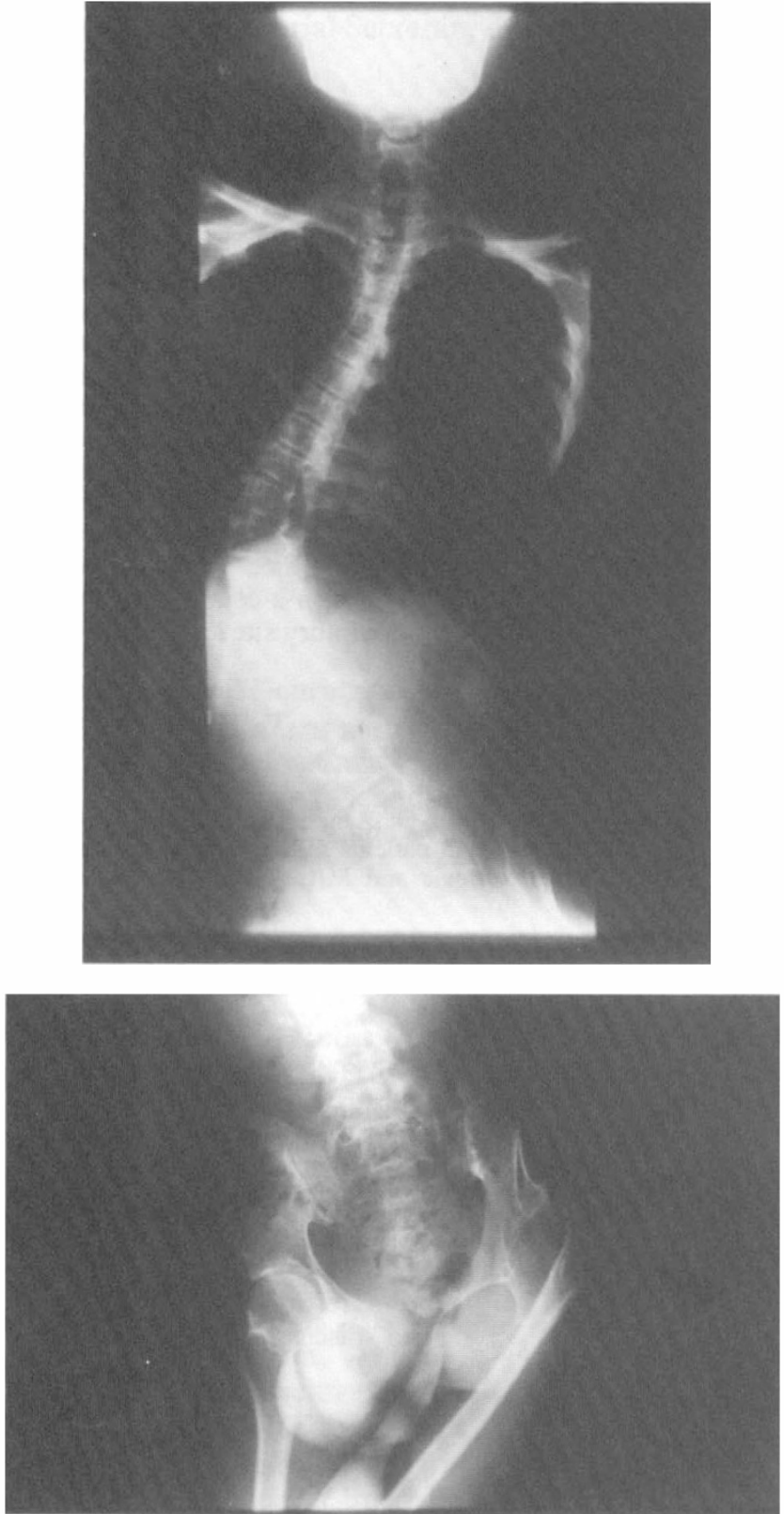

Figure 2 Spinal deformity, dislocation of hip and pelvic tilt revealed at the age of 14

As in the case of adults, it is important to take prophylactic steps in children regarding complications of the SCI, but in children, also, particular attention is focused on the development of spinal deformity and of dislocation of the hip joint. For prophylactic purposes, SCI children are restricted regarding sitting until their vertebral column becomes a reliable support; also, we would recommend a Perthes trolley rather than a wheelchair because then the child can remain prone when moving around. In daily life, they are likely to use a wheelchair more often as they grow older, especially when reaching school age.

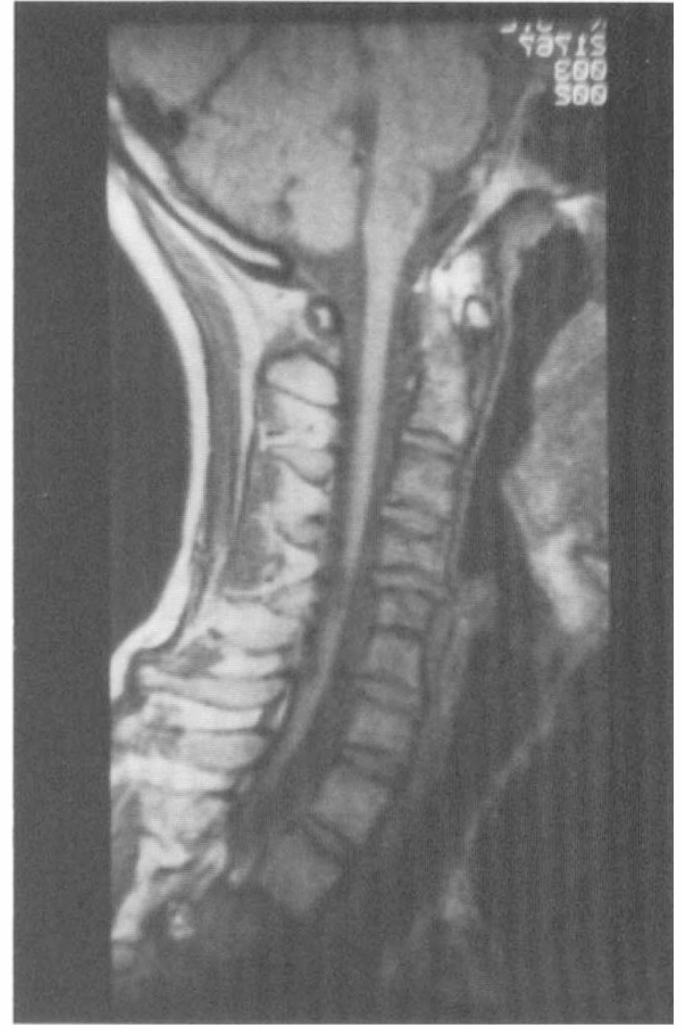

Figure 3 MRI at the age of 18 showed abnormal spinal cord, corpus vertebrae and intervertebral disc

A spinal orthosis and therapeutic exercises for the prevention of the progress of spinal deformity is not always effective. For the treatment of a serious spinal deformity, a surgical operation is required, but there is a risk. It is very difficult to prevent and to treat a spinal deformity resulting from SCI in children, and pressure ulcers and respiratory complications can occur, along with restriction of activities of daily living (ADL).

\section{Conclusion}

In rehabilitating children with spinal cord injuries, it must be remembered that they are in the growing process, and certain serious complications may occur as they grow older. It is important to follow up such children carefully for many years because of possible complications.

\section{References}

1 Allen JP. Spinal cord injury at birth. Handbook of Clinical Neurology 1976; 25: 155-173.

2 Gehrig K, Michaelis LS. Statistics of acute paraplegia and tetraplegia on a national scale. Paraplegia 1968; 6: 93-95.

3 Kewalrmini LS, Orth YS, Kraus JF, Sterling HM. Acute spinal cord lesions in a pediatric population. Paraplegia 1980; 18: 206-219.

4 Burke DC. Traumatic spinal cord paralysis in children. Paraplegia 1974; 11: 268-276. 